\title{
Upper respiratory tract and aural flora of saturation divers
}

\author{
D. M. JONES AND P. DAVIS
}

From the Department of Bacteriology, Withington Hospital, Manchester M20 8LR, UK

SUMMARY The conditions of helium saturation diving promote the proliferation of Gram-negative bacterial species in the external auditory meatus of divers. These changes in flora occurred in the absence of operational diving, that is, no contact with water. The colonising bacteria were autogenous in origin and cross-colonisation was observed between divers. On return to normal atmospheric conditions the aural flora became predominantly Gram-positive again within 48 hours.

The slow return to normal pressures by deep-sea divers, necessary to avoid decompression sickness, restricts the period that can be maintained at depth. This problem is overcome by the saturation diving technique whereby the divers are maintained at high pressure in their living quarters and are transported to the sea bed in a detachable diving bell. The compression chamber and transfer chamber are anchored to the deck of an oil production platform, and the diving bell is detached and winched down to the required depth. On reaching the depth where they are to work, the divers leave the diving bell through a hatch and, on completion of their work, return to the bell, which is then raised to the platform and locked on to the parent compression chamber complex. Other divers in the compression chamber can then transfer to the bell and work can continue underwater in shifts. The deck compression chamber is fitted out with toilets, showers, beds, and life-support systems in general, allowing divers to recuperate between shifts. Food and other items are passed into, or removed from, the chamber through a small pressure-locked transfer port. A team of six divers in such a system could work, rest, and sleep for up to three months at a time in this artificial environment.

The atmosphere within the chambers has a maintained partial pressure of oxygen of around 0.3 to 0.35 atmospheres. The carbon dioxide level is not allowed to exceed 0.005 atmospheres. The pressure of the atmosphere has to be increased to an equivalence of the pressure at which the work has to be performed. The steady partial pressure of oxygen is maintained by adding increasing amounts of helium to the breathing mixture. It is necessary to maintain

Received for publication 5 January 1978 a high ambient temperature $\left(30^{\circ}-35^{\circ} \mathrm{C}\right)$, and the relative humidity is high.

Underwater exploration is a relatively new industry and by its nature is extremely dangerous. It is important that divers remain fit during the conduct of a saturation dive because if a diver becomes unwell the extreme slowness with which decompression can be carried out means that immediate medical attention is not readily available. Among other things, divers may suffer from injuries to the ear, and severe otitis externa has resulted in termination of work schedules. The high humidity and temperature of the environment at saturation lead to hydration of the skin of the external auditory meatus; this favours the proliferation of Gram-negative bacteria, which appear to be the commonest cause of otitis externa among divers (Cobet et al., 1970; Wright and Alexander, 1974).

This study is on the microbial flora of the upper respiratory tract of three divers who performed a simulated dive equivalent to the depth of 1000 feet (305 metres). This 'dive' was undertaken in a new uncontaminated chamber during the proving of a new saturation system. The divers did not, of course, go underwater in the diving bell although they had work tasks to perform to simulate actual working conditions. In this particular 'dive' the diving phase, where there was increasing compression, took 40 hours before the equivalent depth of 305 metres was attained. Conditions at this depth were then maintained for 50 hours and this was followed by a decompression phase which took approximately 12 days.

\section{Material and methods}

Initially, the divers had a rigorous physical exami- 
nation, chest $x$-rays, and spirometry, and they were shown how to take nose, throat, and ear swabs from each other. Nose, throat, and ear swabs were taken on the two days before the dive, daily by the divers themselves after maximum 'depth' had been reached, and follow-up swabs were taken 48 hours and two weeks after the dive had been completed. The swabs taken by the divers were passed out through the transfer port to be plated on to blood agar and MacConkey agar. Blood agar settle plates were put out in various parts of the chamber by the divers on six days of the dive. The plates were removed through the air-lock and left untouched for 30 minutes to allow dissolved helium to escape from the agar. Occasionally the escape of gas was too vigorous and the plates were disrupted. At the conclusion, when the divers left the chamber, environmental samples were taken with swabs and impression cultures. Any Gram-negative bacteria were characterised by routine biochemical methods, and all isolations of Staphylococcus aureus were bacteriophage typed. Proteus strains were compared by the Dienes phenomenon.

\section{Results}

\section{NASAL FLORA}

Diver A was an established nasal carrier of Staph. aureus $(29 / 52+)$, and this was isolated from his nose throughout the period. Diver B was not a staphylococcal carrier initially, but after 10 days in saturation he acquired Staph. aureus $(29 / 52+)$ and continued to carry for the rest of the period of observation, including the post-dive swab. Diver $\mathrm{C}$ carried Staph. aureus $(6 / 42+)$ throughout the study. There was no change in the species of the bacteria forming the normal flora of the anterior nares in any of the three divers during the period of observation.

\section{THROAT FLORA}

The nature of the throat flora of the three divers did not vary during the study.

\section{EXTERNAL AUDITORY MEATUS}

Significant changes in the aural flora of all three divers were observed. Initially, all had a normal, predominantly Gram-positive type of bacterial flora but this changed to predominantly Gram-negative organisms after four to five days in saturation (Fig. 1). Klebsiella oxytocum, Acinetobacter anitratum, Proteus mirabilis, and a non-pigmented Pseudomonas $s p$ were isolated from diver A. Diver B acquired the same strain of Pr. mirabilis (by Dienes) and, in addition, in the right ear, Escherichia coli. Diver C acquired a different strain of $E$. coli but was also colonised by the $E$. coli strain from diver B after 10

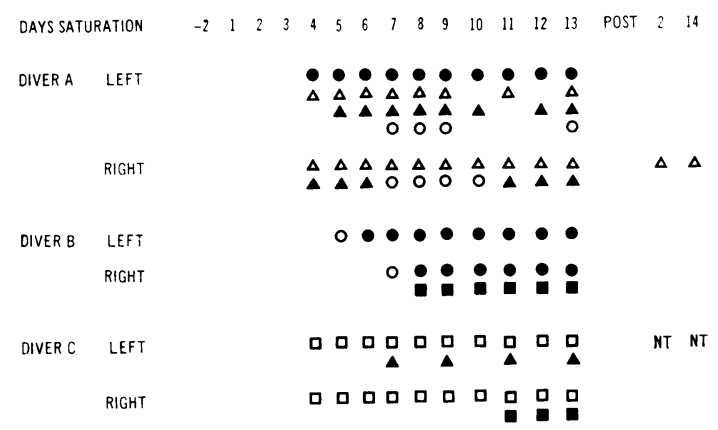

Fig. 1 Gram-negative bacterial species isolated from ears: - Proteus mirabilis; $\triangle$ Klebsiella oxytocum;

$\Delta$ Pseudomonas $s p$; $\bigcirc$ Acinetobacter anitratum;

Escherichia coli (a); $\square$ Escherichia coli $(b)$.

days in saturation. Two days after the saturation dive had been completed the aural flora of two divers was predominantly Gram positive again but $K$. oxytocum was isolated from the right ear of diver A. The findings were similar two weeks later. Diver $\mathrm{C}$ was not available for examination. The divers did not experience symptoms of otitis externa during the simulated dive.

\section{ENVIRONMENTAL STUDIES}

The number of bacteria settling from the air rose throughout the dive (Fig. 2) and most were coagulasenegative staphylococci and micrococci. Gramnegative species were isolated in small numbers and included some of the Gram-negative species isolated from the ears of the divers, for example, $E$. coli, Acinetobacter sp, Pseudomonas sp, and Pr. mirabilis.

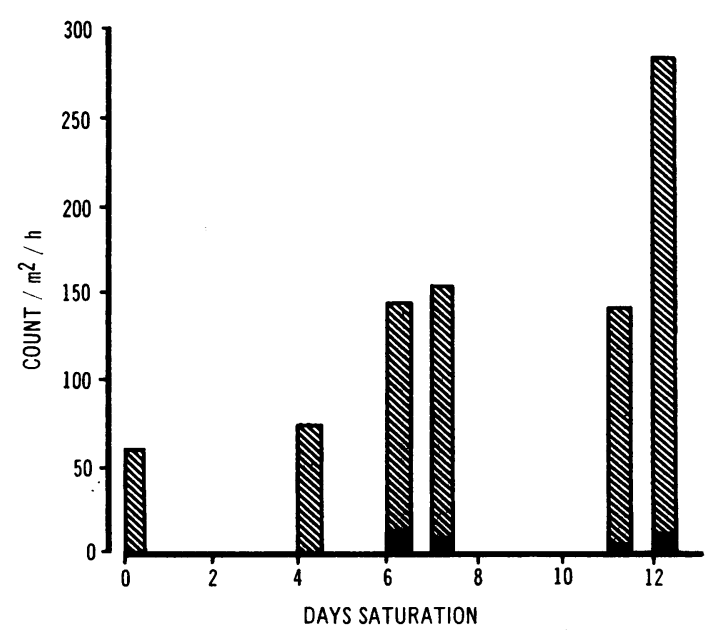

Fig. 2 Settle plate counts during the simulated dive: total colony count; Gram-negative colony count. 
A variety of surfaces within the chamber were sampled at the termination of the dive. Both phage types of Staph. aureus isolated from the divers were recovered, and a small number of colonies of Acinetobacter sp and Pseudomonas sp were also grown. Other organisms similar to those in the divers' ears were not recovered.

\section{Discussion}

This study confirms that in deep saturation dives the high temperature and humidity within the chamber lead to the colonisation of the external auditory meatus with Gram-negative bacteria. The proliferation of Gram-negative bacteria was accompanied by suppression of the normal Gram-positive flora in the meatus. All three divers were affected, and none had Gram-negative organisms in the ears before the dive. The source of the organisms was presumably autogenous but some cross-colonisation was demonstrated later in the period of saturation. Although the same changes have been observed when contact with water was also involved (Wright and Alexander, 1974; Alcock, 1977), our findings, in the absence of any operational diving, confirm that such changes in the aural flora are unavoidably associated with the saturation technique itself. It is this change to a Gram-negative flora in the external auditory meatus that would predispose to the development of otitis externa. There was a rapid reversion to a normal Gram-positive flora once the divers were in ordinary atmospheric conditions, although one diver was still colonised by $K$. oxytocum for at least 14 days. The diving chamber itself showed only a low level of contamination with Gram-negative organisms at the end of the period, and perhaps this is a reflection of the fact that actual diving was not being done. Nevertheless cross-colonisation was seen to occur between the men, and the settle plate studies demonstrated the presence of some of the relevant Gram-negative bacteria in the chamber atmosphere.

\section{References}

Alcock, S. R. (1977). Acute otitis externa in divers working in the North Sea: a microbiological survey of seven saturation dives. Journal of Hygiene, 78, 395-409.

Cobet, A. B., Wright, D. N., and Warren, P. I. (1970). Tektite-I program: bacteriological aspects. Aerospace Medicine, 41, 611-616.

Wright, D. N., and Alexander, J. M. (1974). Effect of water on the bacterial flora of swimmers' ears. Archives of Otolaryngology, 99, 15-18.

Requests for reprints to: Dr D. M. Jones, Public Health Laboratory, Withington Hospital, Manchester, M20 8LR. 